Supplement of Saf. Nucl. Waste Disposal, 1, 117-119, 2021

https://doi.org/10.5194/sand-1-117-2021-supplement

(C) Author(s) 2021. CC BY 4.0 License.

(c) (i)

Supplement of

\title{
Pilot plant tests to demonstrate the functionality of sealing elements made of salt cut bricks
}

Uwe Düsterloh et al.

Correspondence to: Uwe Düsterloh (uwe.duesterloh@tu-clausthal.de)

The copyright of individual parts of the supplement might differ from the article licence. 


\section{Pilot plant tests to demonstrate the functionality of sealing elements made of salt cut bricks}

1. Motivation: initial porosity of chrushed salt is given by some $35-40 \%$


initial porosity of salt cut bricks is given by some $1-5 \%$ depending on joint thickness and brick size
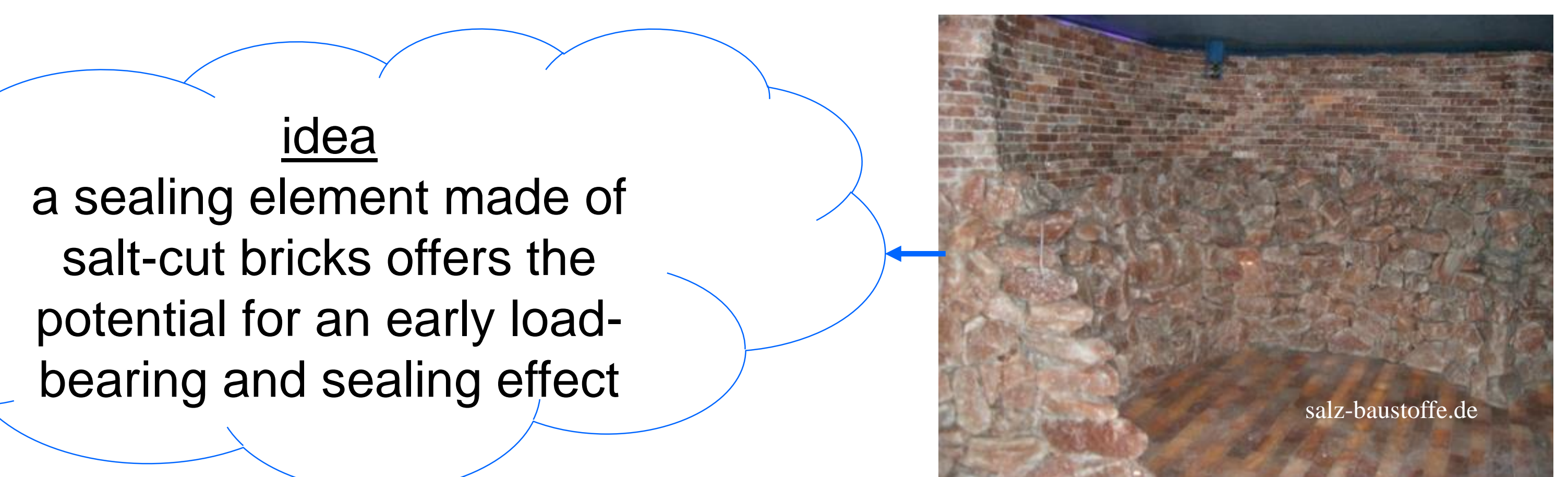

2. Preliminary investigations into the sealing behaviour of salt cut surfaces

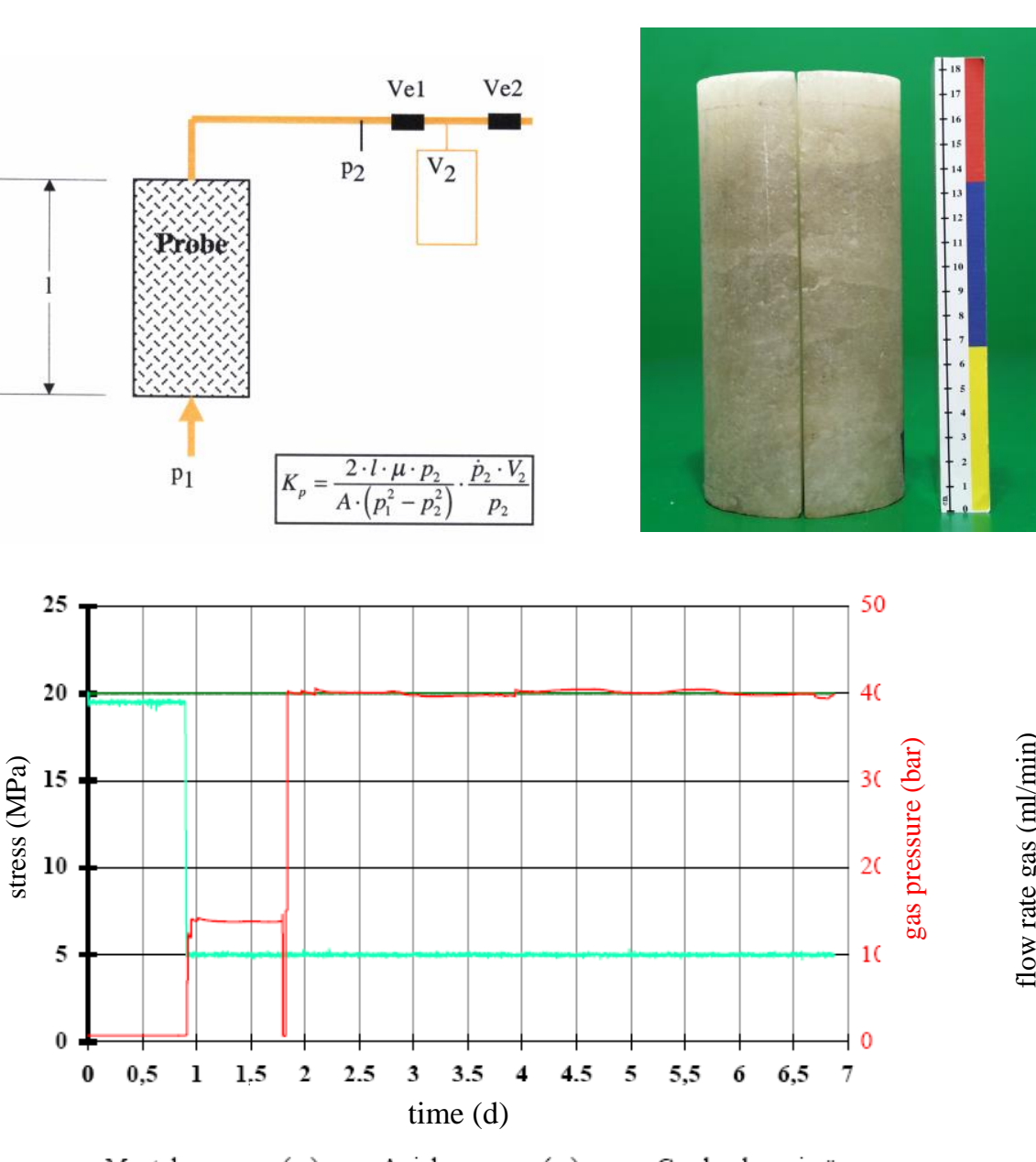

moistered cut surface
dry $\mathrm{N}_{2}$ gas

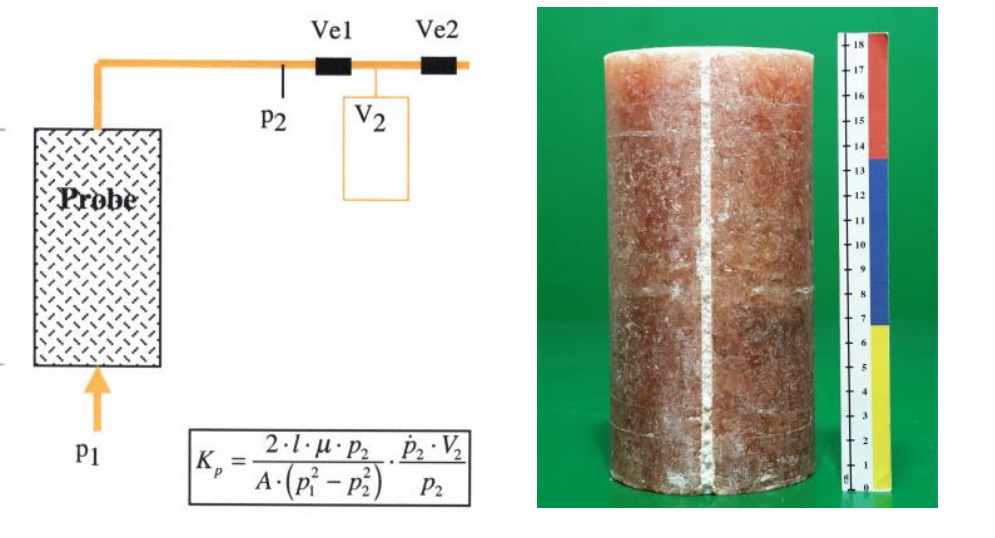

cul surtace
wet $N_{2}$ gas
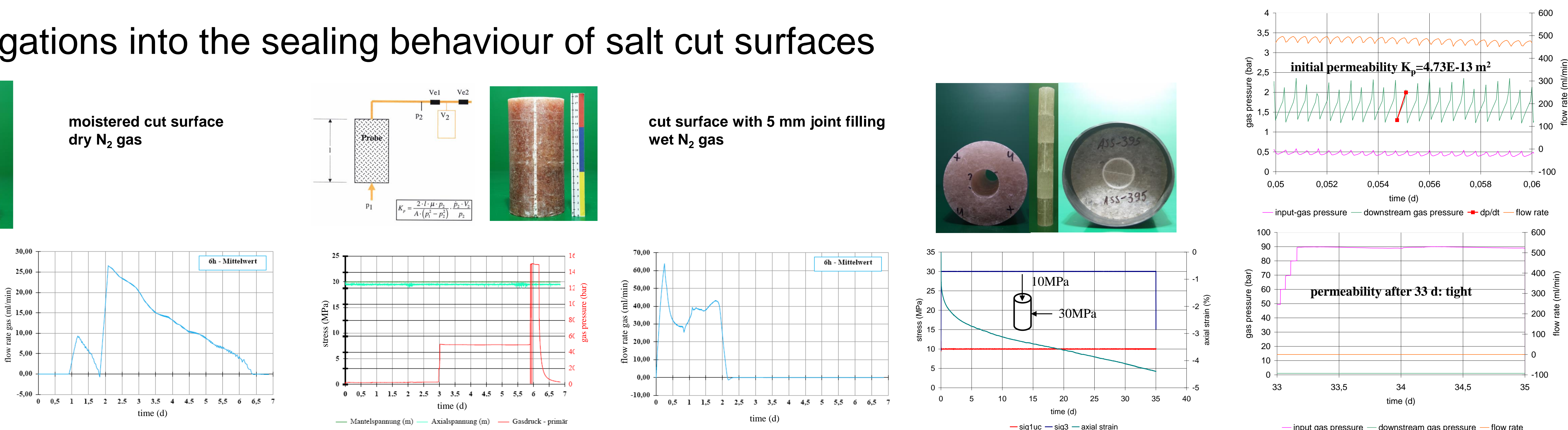

3. Construction of a pilot plant to analyze in detail the performance of a sealing element made of salt cut bricks
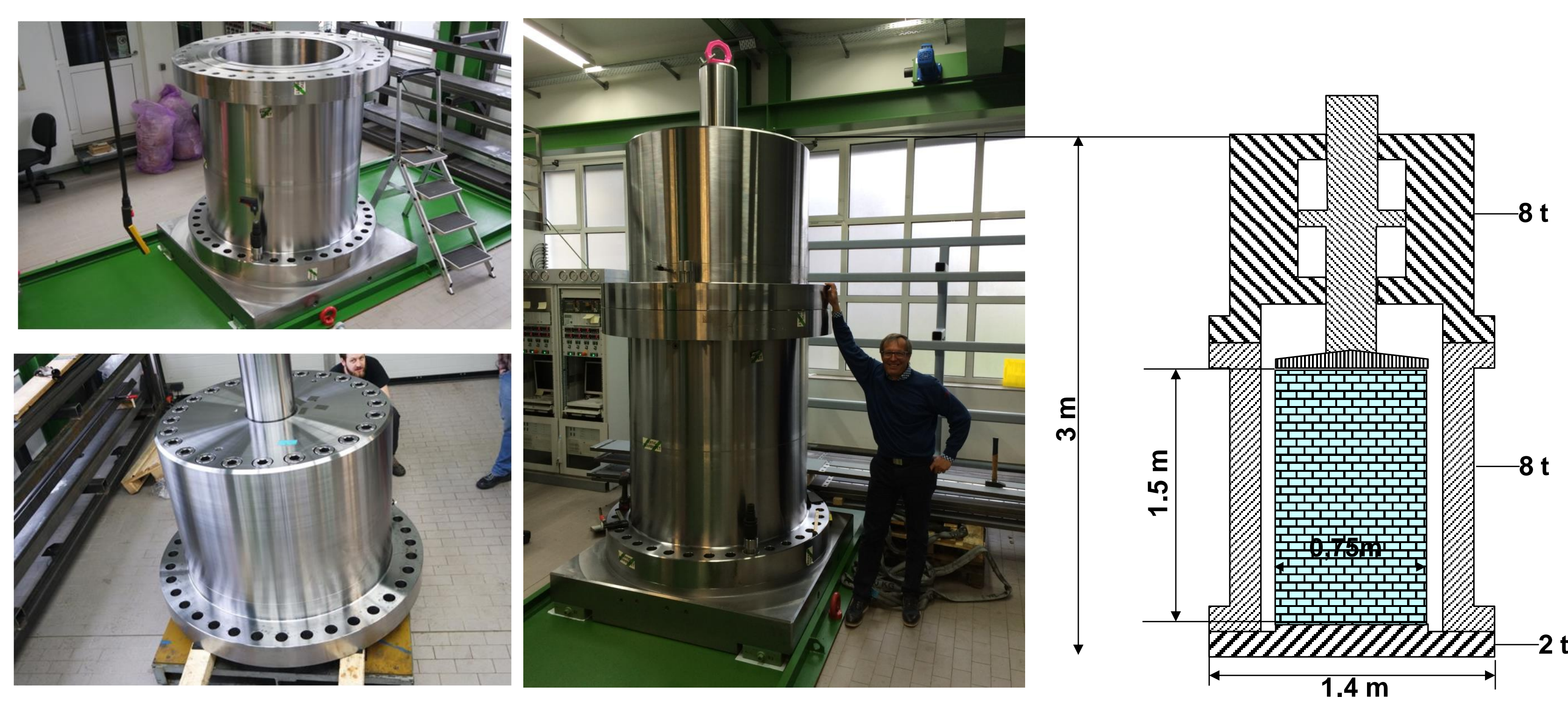

Performance data:
$\max _{3}=25 \mathrm{MPa}$
$\operatorname{maxF}_{a x}=28 \mathrm{MN}$
$\operatorname{maxP}_{\text {gas-primär }}=25 \mathrm{MPa}$
$\max P_{\text {gas-sekundär }}=25 \mathrm{MPa}$
$\operatorname{maxT}=80^{\circ} \mathrm{C}$
$\max \Delta \mathrm{u}= \pm 30 \mathrm{~cm}$
sample size: $1,5 \mathrm{~m} \times 0,75 \mathrm{~m}$

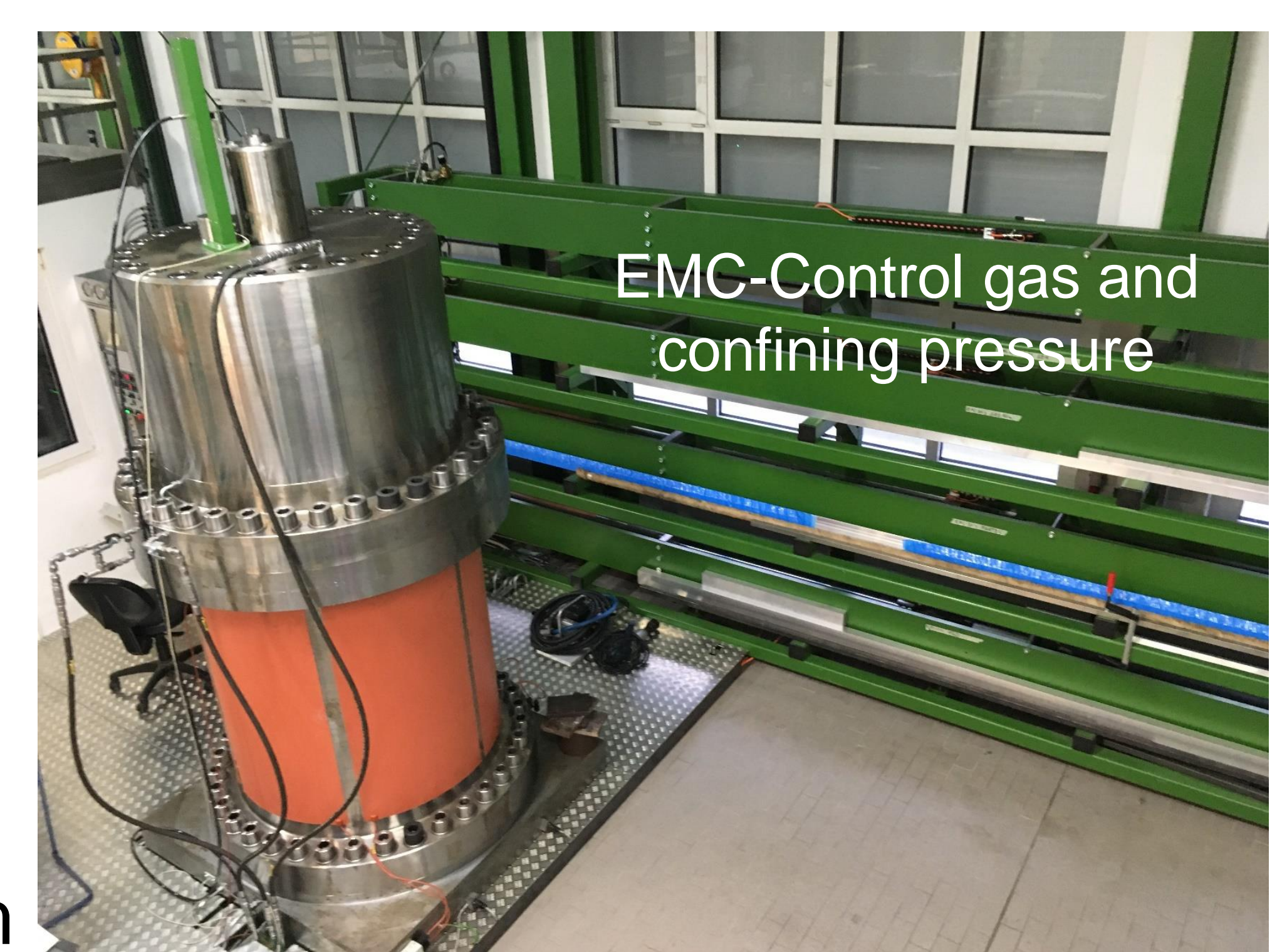

4. Production of salt cut bricks - avoidance of axially continuous joints


$12 \mathrm{t}$ basic raw material $\rightarrow 0,66 \mathrm{~m}^{3} / 1,5 \mathrm{t}$ sealing element $\approx 6 \mathrm{t}$ crushed salt

$\approx 4,5 \mathrm{t}$ offcuts

$\approx 7$ salt bricks per week $\rightarrow \approx 1$ a per sample $\rightarrow 375$ bricks per sample

5. Triaxial THM test control and evaluation

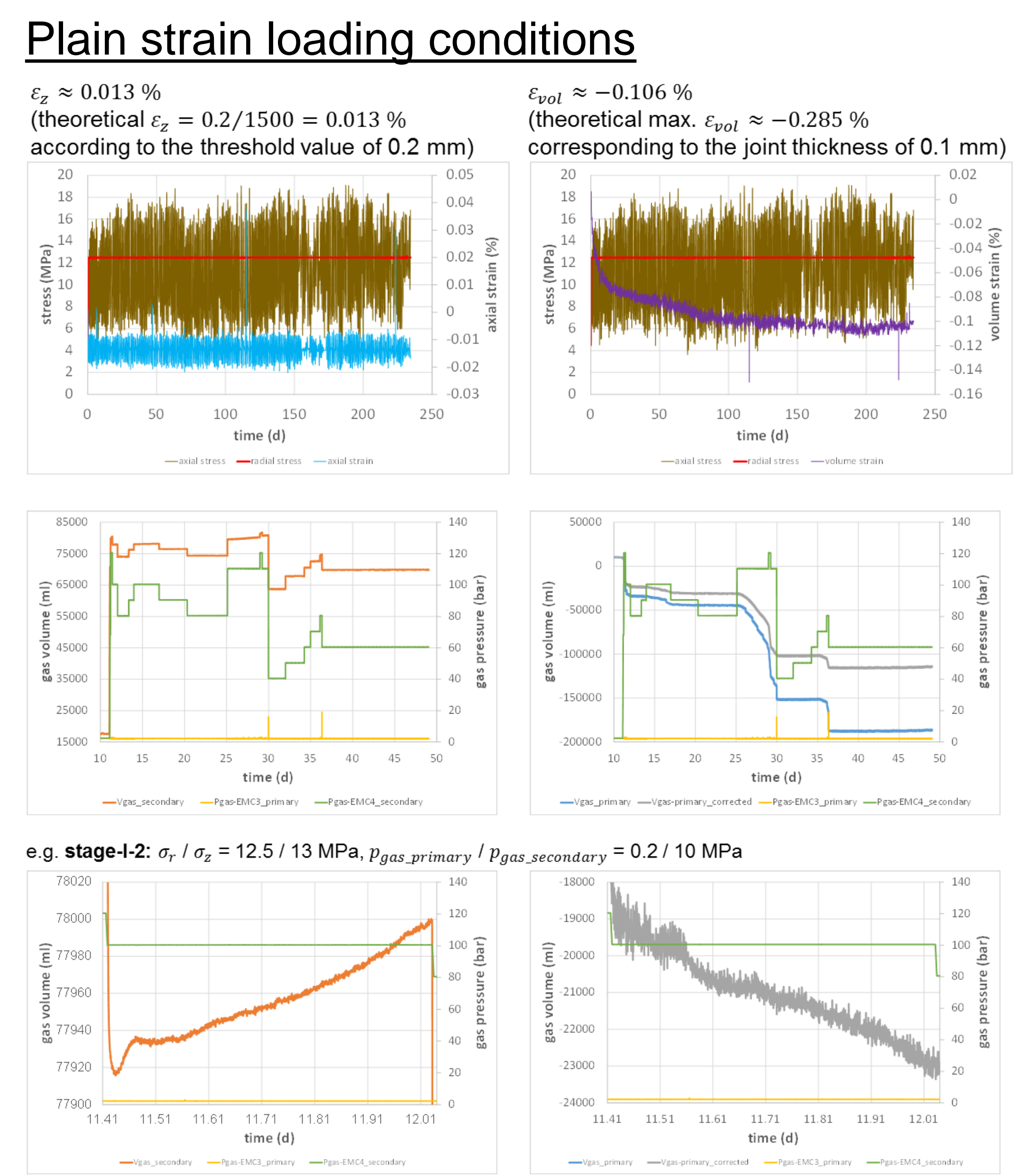

Permeability calculation
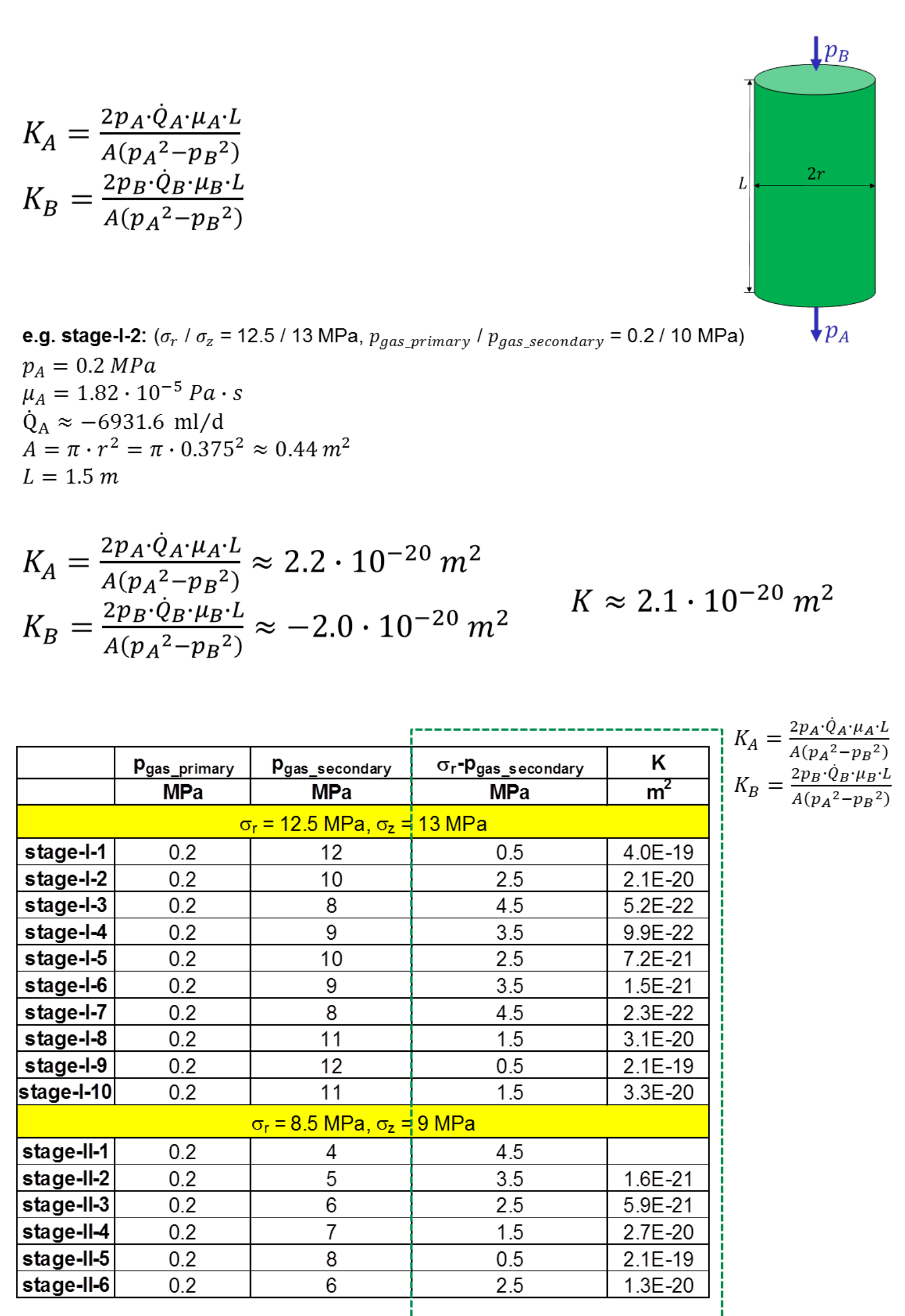

Measurement results:

a sealing element made of salt-cut bricks with moistened cut surfaces achieves a high sealing effect under typical in situ loading conditions within a few months

$1 \mathrm{E}-18$



$1 \mathrm{E}-22$ 